\title{
Correction to: Cerebrospinal fluid amyloid- $\beta$ 2-42 is decreased in Alzheimer's, but not in frontotemporal dementia
}

\author{
Mirko Bibl ${ }^{1,2} \cdot$ Marion Gallus ${ }^{3} \cdot$ Volker Welge $^{3} \cdot$ Hermann Esselmann $^{4} \cdot$ Stefanie Wolf $^{1} \cdot$ Eckart Rüther $^{5}$. \\ Jens Wiltfang ${ }^{4,6}$
}

Published online: 29 August 2018

(c) Springer-Verlag GmbH Austria, part of Springer Nature 2018

\section{Correction to: J Neural Transm (2012) 119:805-813 https://doi.org/10.1007/s00702-012-0801-3}

The respective first and last authors of this article, Mirko Bibl and Jens Wiltfang, would like to clarify the issue of the seeming duplicate publication of a figure in two articles.

From E-mails dated 14th June 2018 (Dr. Kalvodova) and 15th June 2018 (Dr. Solomon) we understood that a reader has notified you of similarities of Fig. 4 in Proteomics Clinical Applications in the publication https://doi.org/10.1002/ prca.201100082 (1) with Fig. 1 in https://doi.org/10.1007/ s00702-012-0801-3 in Journal of Neural Transmission (2).

While the historically first paper (received: 19.09.2011; revised: 16.10.11; accepted: 25.10.11; first published: 25.04.2012) published in Proteomics Clinical Applications

The original article can be found online at https://doi.org/10.1007/ s00702-012-0801-3.

Mirko Bibl

m.bibl@kliniken-essen-mitte.de;

prof-bibl@seelische-gesundheit-mettmann.de

Jens Wiltfang

Jens.Wiltfang@med.uni-goettingen.de

1 Department of Psychiatry, University of Goettingen, von-Siebold-Str. 5, 37075 Goettingen, Germany

2 Present Address: Praxis für ärztliche Psychotherapie, Gartenstrasse 2, 40822 Mettmann, Germany

3 Department of Psychiatry, Psychotherapy and Addiction Medicine, Kliniken Essen-Mitte, University of Duisburg-Essen, Henricistrasse 92, 45136 Essen, Germany

4 Department of Psychiatry and Psychotherapy, University of Duisburg-Essen, 45147 Essen, Germany

5 Department of Psychiatry and Psychotherapy, Ludwig-Maximilian-University of Munich, Geschwister-Scholl-Platz 1, 80539 Munich, Germany

6 Present Address: Clinic of Psychiatry and Psychotherapy, University Medical Center, Von-Siebold-Strasse 5, 37075 Göttingen, Germany describes the methodological details of the characterization of the aminoterminally truncated and oxidized Abeta-peptide species in Cerebrospinal Fluid (CSF) the second paper, (received: 21.01.2012; accepted: 22.3.2012; first online: 19.4.2012) published in Journal of Neural Transmission aimed to explore the potential usefulness of their quantification in the clinical diagnostics of dementia. Consequently, different results were published in the two journals.

Indeed, these two figures in question derive from one and the same master blot. However, in our opinion, we cited this figure in the second paper (Journal of Neural Transmission) in a fully correct way, namely as follows:

- In the second paper (Journal of Neural Transmission) we stated in the results chapter on p. 807:

"Based on a previously published sequential aminoterminally and carboxyterminally specific immunoprecipitation protocol followed by subsequent Ab-SDS-PAGE/ immunoblot analysis, the CSF concentrations of the $\mathrm{Ab}$ peptides 1-37, 1-38, 1-38ox, 1-39, 1-40, 1-40ox, 1-42, 2-40 and 2-42 could be analyzed in the groups NDC, AD and FTD (Fig. 1)."

- We have cited the historically first paper published in $J$ Proteomics Clin. Appl., i.e. in Journal of Neural Transmission, two times in our second publication, namely at the end of the left column of p. 806 and at the top of the left column of p. 810 .

A second version of the same master blot modified as compared to the first paper (J Proteomics Clin. Appl.) was shown only for didactic reasons that is the prototypic illustration of the Abeta-peptide separation by urea based western immunoblot. Thus, the reason for showing the figure again in the second publication was solely to improve the intelligibility of the paper. 
The reason for cutting and flipping the blot in the second publication also resulted from our attempts to improve its intelligibility.

In detail, by cutting the blot's original size we attempted to highlight its most relevant areas. In the first publication, the master blot was flipped in order to put labels, i.e. the names of peptides, on the left side of the blot and next to standard peptide lanes. Labels were put outside the blot to not disturb the visibility of blot bands and facilitate the visual comparability of their heights. In the second publication the figure served only for illustration so that peptide labels were integrated in the blot picture. In both publications, we aimed to stick to the convention that labels of blot bands are put on the left side of blots.

In light of these considerations, we respectfully disagree with the opinion of having performed ethical or scientific misconduct and thus reject the accusation raised by a notnamed reader of having edited the blot with the intention to hide its mutual duplicate publication.

The original version of this article does not reflect the current affiliations of Mirko Bibl and Jens Wilfang.

Their current affiliations are as follows:
Mirko Bibl

Praxis für ärztliche Psychotherapie, Gartenstrasse 2, 40822 Mettmann, Germany

prof-bibl@seelische-gesundheit-mettmann.de

Jens Wilfang

University Medical Center, Clinic of Psychiatry and Psychotherapy, Director, Von-Siebold-Strasse 5, 37075 Göttingen, Germany

Jens.Wiltfang@med.uni-goettingen.de

\section{References}

1. Bibl M, Gallus M, Welge V, Lehmann S, Sparbier K, Esselmann H, Wiltfang J (2012a) Characterization of cerebrospinal fluid aminoterminally truncated and oxidized amyloid- $\beta$ peptides. Proteom Clin Appl 6(3-4):163-169. https://doi.org/10.1002/prca.201100082

2. Bibl M, Gallus M, Welge V, Esselmann H, Wolf S, Rüther E, Wiltfang $J$ (2012b) Cerebrospinal fluid amyloid- $\beta 2-42$ is decreased in Alzheimer's, but not in frontotemporal dementia. J Neural Transm (Vienna) 119(7):805-813. https://doi.org/10.1007/s00702-0120801-3 (Epub 19 Apr 2012) 